\title{
Analysis of a Sound Signal for Quality Monitoring in Laser Microlap Welding
}

\author{
Bo-Si Kuo and Ming-Chyuan Lu * \\ Department of Mechanical Engineering, National Chung Hsing University, 145 Xingda Rd., South Dist., \\ Taichung 402, Taiwan; stanscreechkk@gmail.com \\ * Correspondence: mclu@dragon.nchu.edu.tw
}

Received: 15 February 2020; Accepted: 3 March 2020; Published: 12 March 2020

\begin{abstract}
This study focused on correlation analysis between welding quality and sound-signal features collected during microlaser welding. The study provides promising features for developing a monitoring system that detects low joint strength caused by a gap between metal sheets after welding. To obtain sound signals for signal analysis and develop the monitoring system, experiments for laser microlap welding were conducted on a laser microwelding platform by installing a microelectromechanical system (MEMS) microphone away from the welding point, and an acoustic emission (AE) sensor on the fixture. The gap between two metal sheet layers was controlled using clamp force, a pressing bar, and the appropriate installation of a thin piece of paper between the metal sheets. After sound signals from the microphone were collected, the correlation between features of time-domain sound signals and of welding quality was analyzed by categorizing the referred signals into eight sections during welding. After appropriately generating the features after signal analysis and selecting the most promising features for low-joint-strength monitoring on the basis of scatter index J, a hidden Markov model (HMM)-based classifier was applied to evaluate the performance of the selected sound-signal features. Results revealed that three sound-signal features were closely related to joint-strength variation caused by the gap between two metal-sheet layers: (1) the root-mean-square (RMS) value of the first section of sound signals, (2) the standard deviation of the first section of sound signals, and (3) the standard deviation to the RMS ratio of the second section of sound signals. In system evaluation, a 100\% classification rate was obtained for normal and low-bonding-strength monitoring when the HMM-based classifier was developed on the basis of the three selected features.
\end{abstract}

Keywords: laser microwelding; quality monitoring; audible sound

\section{Introduction}

Laser welding is a key technology that has been used for decades to fuse various components, ensuring a low heat-affected zone on components during manufacturing. Due to the continuous development of the fiber laser system and the reduction of its operational costs, laser microwelding has been utilized in various applications throughout the past decade. However, the lack of a quality-monitoring system that confirms joint quality can limit the application of laser microwelding to some products that require high reliability. In laser microlap welding, the gap between two metal-sheet layers increases the energy required for joint formation, thus reducing joint quality. Therefore, a gap or quality-monitoring system is crucial for verifying welding quality, and for extending its industrial applications to more fields in the industry.

Some studies have reported the monitoring of welding conditions during welding processes. However, most studies focused on keyhole-mode laser welding with the conventional macroscale of welding. Shao et al. [1] reviewed various types of sensors adopted for keyhole-mode laser welding, 
such as audible-sound, acoustic-emission (AE), and optical sensors. An audible sound sensor has the advantage of easy installation, and sound-signal generation is closely correlated to weld-pool generation. Smith [2] proposed an analytical model to develop a relationship between audible sound and weld-pool oscillation. Sun [3] studied a correlation between sound-signal features and defects generated during keyhole-mode welding. Shimada et al. [4] confirmed that the energy level of a sound signal generated through welding has a close relationship with laser-power density and welding-penetration condition. Some studies [5-8] considered frequency-domain sound signals as potential features to identify defects in the arc-welding quality. Luo et al. used audible sound signals for conduction-mode welding to identify the differences between keyhole and conduction-mode welding [9]. This study also discusses the effect of the gap between two metal sheets and plate orientation on sound-signal features. To study systems that monitor the quality of keyhole-mode welding, we developed a sound-based quality-monitoring system based on a neural network [10-12]. Moreover, Sun [3] and Jin et al. [13] developed sensor-fusion monitoring systems by integrating sound signals with other AE or optical signals. Noise is a problem encountered while applying a sound signal to monitor welded-joint quality. A noise-reduction method was proposed by Huang et al. [14] to reduce the noise effect and to ensure that sound signals can be used in monitoring joint quality during laser welding.

Few studies conducted welded-joint quality monitoring in conduction-mode welding. Chien et al. [15] developed a quality-monitoring system on the basis of audible-sound signals for thin-plate butt welding. The present study focused on analysis of the correlation between the low bonding strength of welding and sound-signal features. A hidden Markov model (HMM)-based monitoring system was applied to evaluate the quality-monitoring performance of the selected sound features.

\section{Experiment Setup}

\subsection{Equipment and Sensors}

To analyze sound signals obtained from laser microlap welding of joints with various qualities, a number of experiments were conducted on a laser-microwelding research platform integrated with a microelectromechanical system microphone (SPM0408LE5H-TB, Knowles, Itasca, IL, USA) that had a frequency range of $100-10 \mathrm{kHz}$, and an AE sensor (8152B121, Kistler, Winterthur, Switzerland) that had a frequency range of 50-400 kHz. For data acquisition, a PCI NI 6132 was used to collect AE and sound signals at a sampling rate of $2 \mathrm{MHz}$.

\subsection{Experiment Design}

Welding parameters in this study are listed in Table 1 . To quantify the joint condition in microlap welding, a peeling test was conducted for each welded sample. Moreover, peeling force was obtained by breaking the joint with self-designed equipment, as displayed in Figure 1. The peeling force required to break the joint was measured using a Kistler 9217A load cell, and the level was recorded during the entire testing process.

Table 1. Welding parameters.

\begin{tabular}{cc}
\hline Laser Power(W) & 105 \\
\hline Scan Speed $(\mathrm{mm} / \mathrm{s})$ & 200 \\
\hline Pulse Frequency (kHz) & 0.01 \\
\hline Processing Time (ms) & 2 \\
\hline
\end{tabular}




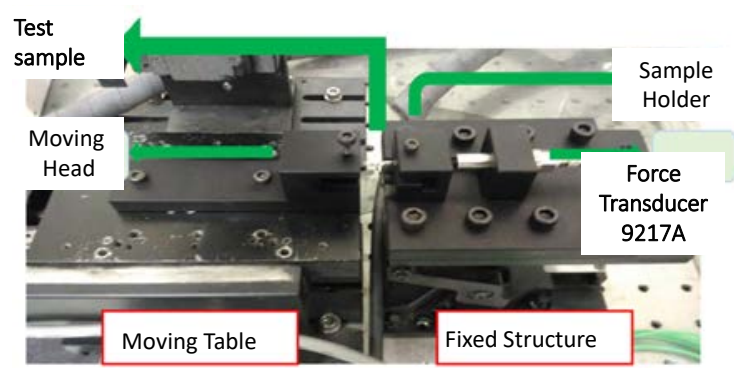

Figure 1. Peeling test.

In a practical welding situation, the loss of appropriate contact between two layers of welded metal sheets might be due to the deformation of thin workpieces or the lack of clamping force caused by improper clamper design. Partial loss in contact reduces joint strength or does not form a joint. To simulate the aforementioned conditions, welding location, the implementation of a central clamp, and the installation of thin paper between two metal-sheet layers were controlled to generate different contact conditions between the metal-sheet layers. Welding quality was then confirmed by conducting the peeling test before signal analysis. Figure 2 displays the fixture design used for generating proper and improper contacts. To generate improper contact between two metal sheets, the central clamp bar was removed, and low-value torque was applied to the screw. Moreover, the welding location was chosen to be close to the central part of the fixture, and a paper sheet that did not cover the welding point was installed between the metal sheets to increase the chance of gap generation. Conversely, to generate proper contact between the metal sheets, the welding location was moved close to the clamp screw, high-value screw torque was applied, and a central clamp bar was implemented. The summary of torque and setup is presented in Table 2.

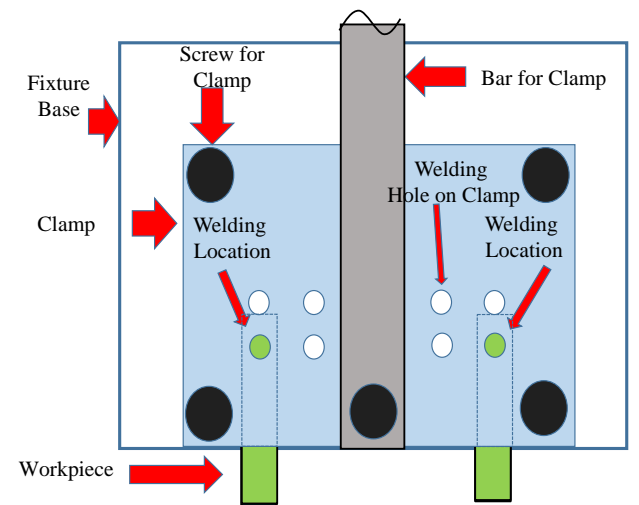

(a)

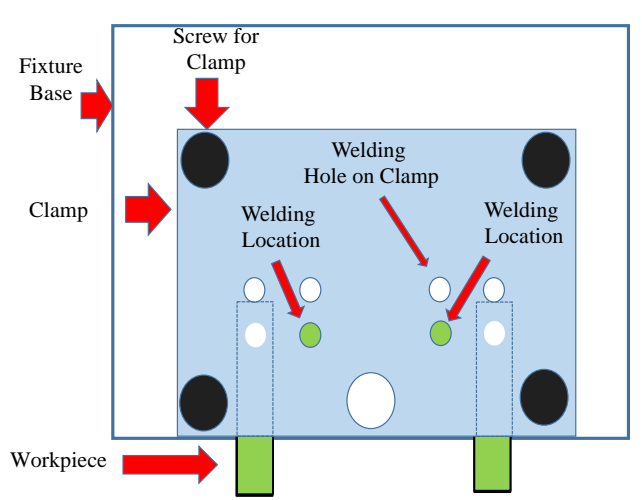

(b)

Figure 2. Setup for various contact conditions during welding: (a) proper contact; (b) contact with a gap between metal sheet layers.

Table 2. Parameter design for generating proper and improper contacts.

\begin{tabular}{ccc}
\hline & Proper Contact & Loss of Contact \\
\hline Torque for screw & $6 \mathrm{~N}$ & $1.5 \mathrm{~N}$ \\
\hline Extra Central Clamp & Yes & No \\
\hline Thin paper between workpiece & No & Yes \\
\hline Welding Location & Close to the corner of clamp & Close to central Line \\
\hline
\end{tabular}




\section{System Development and Verification}

Scatter index $J=\frac{R_{c}(k, k)}{R(k, k)}$ that estimates the between-class to within-class scatter ratio was used to analyze the correlation between selected features and bonding quality. Between-class scatter $R_{c}$ and within-class scatter $R$ are defined as follows [16]. The feature mean for each class $\bar{Y}_{i}(k)$ was obtained using individual features $Y_{i j}(k)$ :

$$
\bar{Y}_{i}(k)=\frac{1}{M_{i}} \sum_{j=1}^{M_{i}} Y_{i j}(k)
$$

where

$$
\begin{aligned}
& i=i^{\text {th }} \text { class } \\
& j=j^{\text {th }} \text { pattern in a class } \\
& k=k^{\text {th }} \text { feature } \\
& M_{i}=\text { the number of pattern in class } C_{i} .
\end{aligned}
$$

The overall system mean $\bar{Y}$ is determined as follows:

$$
\bar{Y}(k)=\sum_{i=1}^{C} p_{i} \bar{Y}_{i}(k)
$$

where

$p_{i}=$ a priori probability of class $C_{i}$

$C=$ number of classes.

Within-class scatter is obtained by calculating covariance for each feature as follows:

$$
R_{i}(k)=\frac{1}{M_{i}} \sum_{j=1}^{M_{i}}\left(Y_{i j}(k)-\bar{Y}_{i j}(k)\right)\left(Y_{i j}(k)-\bar{Y}_{i j}(k)\right)^{T}
$$

Moreover, the individual-class scatter is defined as follows:

$$
R_{c}(k)=\sum_{i=1}^{C} p_{i}\left(\bar{Y}_{i}(k)-\bar{Y}(k)\right)\left(\bar{Y}_{i}(k)-\bar{Y}(k)\right)^{T}
$$

From Equations (3) and (4), the feature-selection criterion, a cost function, is defined as follows:

$$
J(k)=\frac{R_{c}(k)}{R(k)}
$$

where $R(k)=\sum_{i=1}^{C} R_{i}(k)$.

The HMMs for determining welding quality were developed using collected training sound signals. Once the models were developed, the unknown condition of welding quality was determined using collected sound signals and developed models with selected features. The schematic of determining the unknown quality condition by using the developed model is illustrated in Figure 3. In this method, signals collected from events other than those used for model development served as input data. Moreover, quality state $S_{n}$ based on selected features was determined by referring to the model with a large probability value based on the Viterbi algorithm. By using a combination of results obtained from various selected features, the final decision pertaining to the quality state was determined by fusing all decisions. 


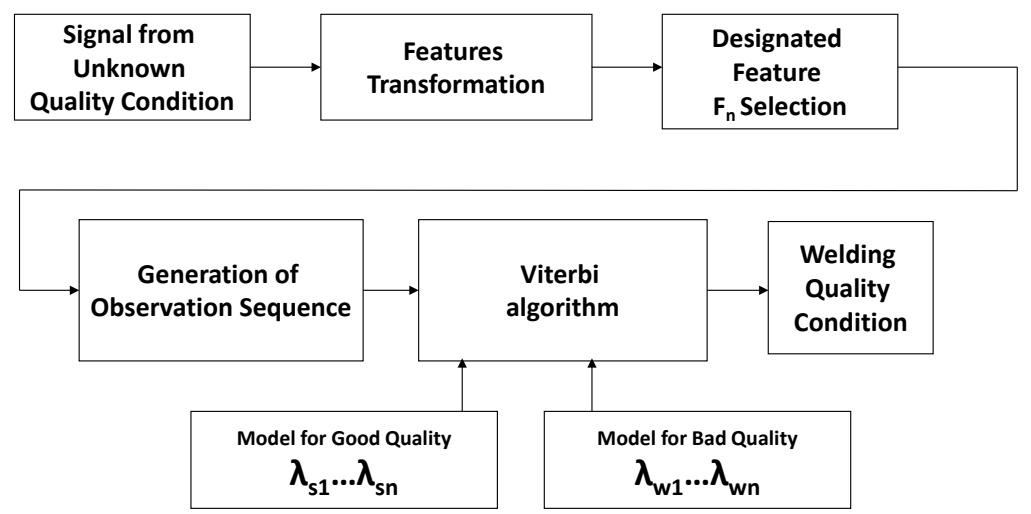

Figure 3. Schematic of determining unknown quality condition by using developed model [17].

\section{Results and Discussion}

To verify the joint strength of each welded sample, a peeling test was conducted. Moreover, the surface condition of a broken joint was examined using an optical microscope. Surface conditions pertaining to the top and bottom surfaces of both metal-sheet layers after the peeling test are shown in Figure 4. The surface condition presented in Figure $4 \mathrm{a}$ was obtained from the case in which the peeling force was higher than $15 \mathrm{~N}$, and that involved proper contact between layers. The surface condition presented in Figure $4 \mathrm{~b}$ was obtained from the case with very low peeling force that involved improper contact between layers. The clear tearing of the material on the bottom layer is shown in Figure 4a, and the separated part of the material was observed to be joined together on the bottom surface of the top layer of the sample. Conversely, no material separation can be observed in Figure $4 \mathrm{~b}$ from the bottom layer of the sample. Only heat-effect zoom was observed at the bottom surface of the top layer and the top surface of the bottom layer. This finding suggests that no significant joint was created between the two metal-sheet layers. Peeling forces in the proper contact cases were in the range of $12-17 \mathrm{~N}$ in this study. No peeling force was available for low-strength-bonding cases because complete peeling occurred abruptly when the peeling test was implemented.

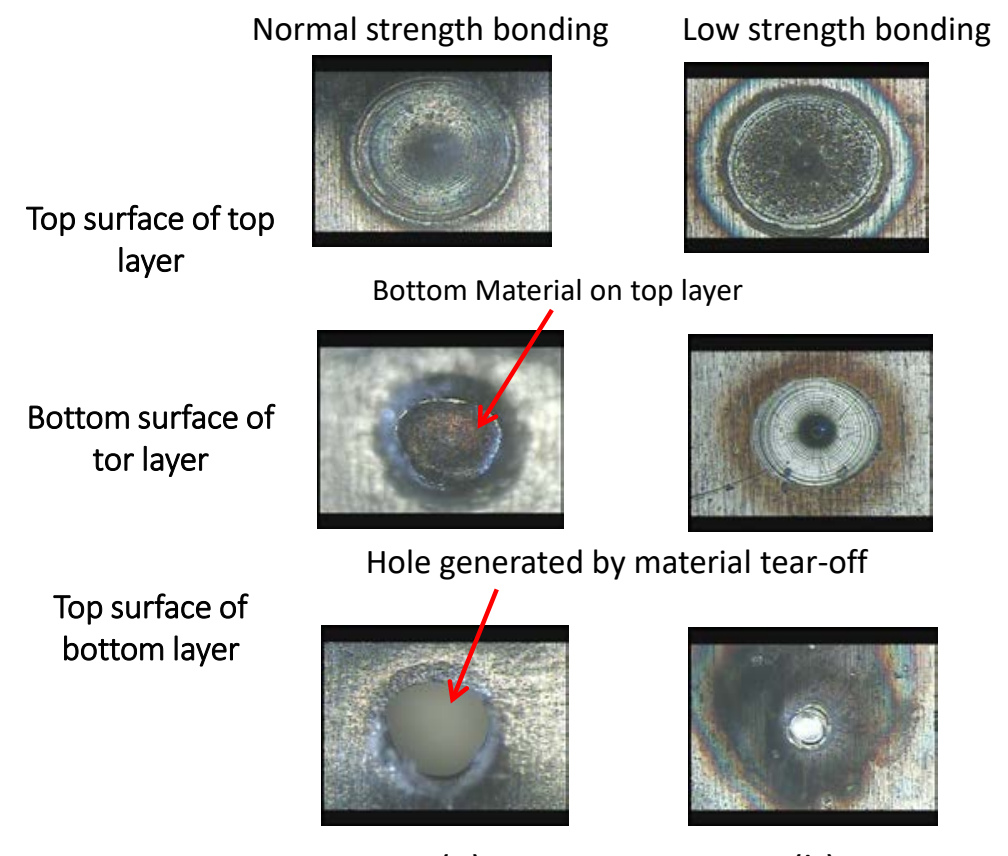

(a)

(b)

Figure 4. Surface condition of samples after peeling test: (a) high joint strength; (b) low joint strength. 
Because the AE signal was generated immediately when the laser beam and samples came into contact, AE signals were collected simultaneously with audible sound (Figure 5) to verify the starting point of the laser application to the metal sheets. Both $\mathrm{AE}$ and sound signals presented different characteristics when welding was conducted. Sound signals collected from cases with normal and low-joint-bonding strengths are shown in Figure 6. Different patterns were observed between the two cases (Figure 6a,c), and signal energy seemed to be a good feature to identify the case with low-bonding-strength joint. However, by reviewing the sound-signal pattern obtained from 20 samples in the case with normal-joint-bonding strength, and 20 samples in the case with low-joint-bonding strength, the variation of signal energy in the same case was observed. Low signal energy was observed for some samples with high-joint-bonding strength (Figure 6b). On the other hand, high signal energy was observed for some samples with low-joint-bonding strength (Figure 6d). After calculating the root-mean-square (RMS) values of full-length sound signals for 20 samples in the normal-joint-bonding strength case, and 20 samples in the low-joint-bonding strength case (Figure 7), a mixture zone of RMS values of full-length sound signals for both cases was observed. Therefore, by only adopting RMS values of full-length sound signals as the feature in monitoring-system development, this may lead to failure in identifying joint-bonding strength. Based on above discussions, other signal features should be studied and extracted to improve system robustness in monitoring joint-bonding strength in laser microlap welding.

(a)

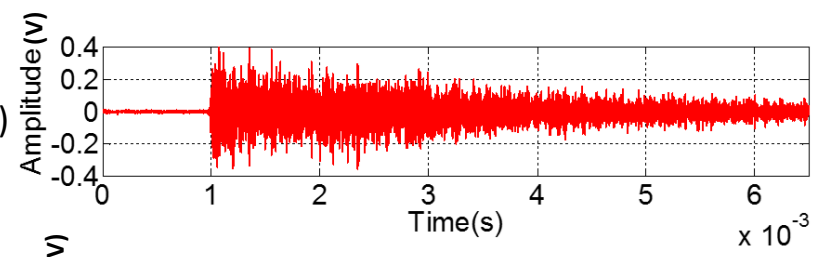

(b)

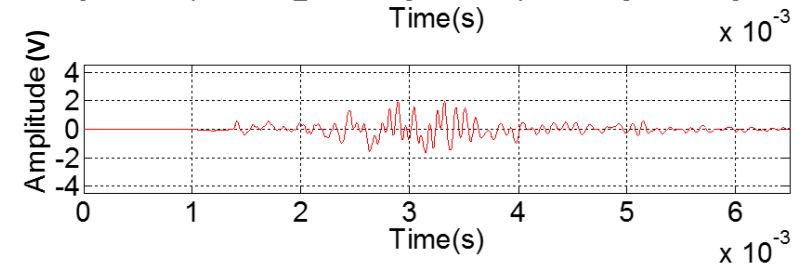

Figure 5. Signals collected during laser microwelding: (a) acoustic-emission (AE) signal; (b) audible sound.

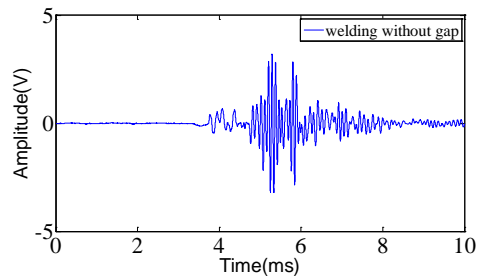

(a)

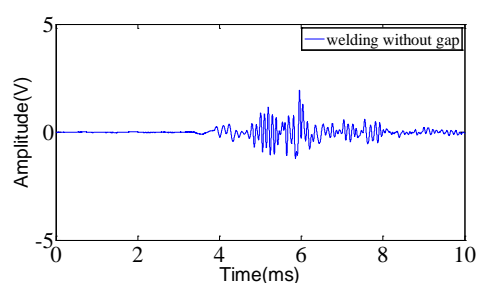

(b)

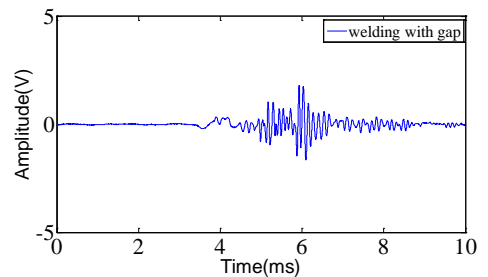

(c)

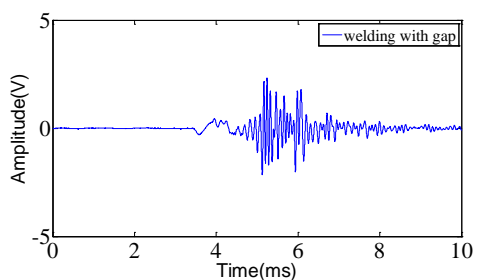

(d)

Figure 6. Sound signals collected during laser microlap welding: (a) normal joint strength-Nsample 1; (b) normal joint strength-Nsample 2; (c) low joint strength-Lsample 1; (d) low joint strength-Lsample 2. 


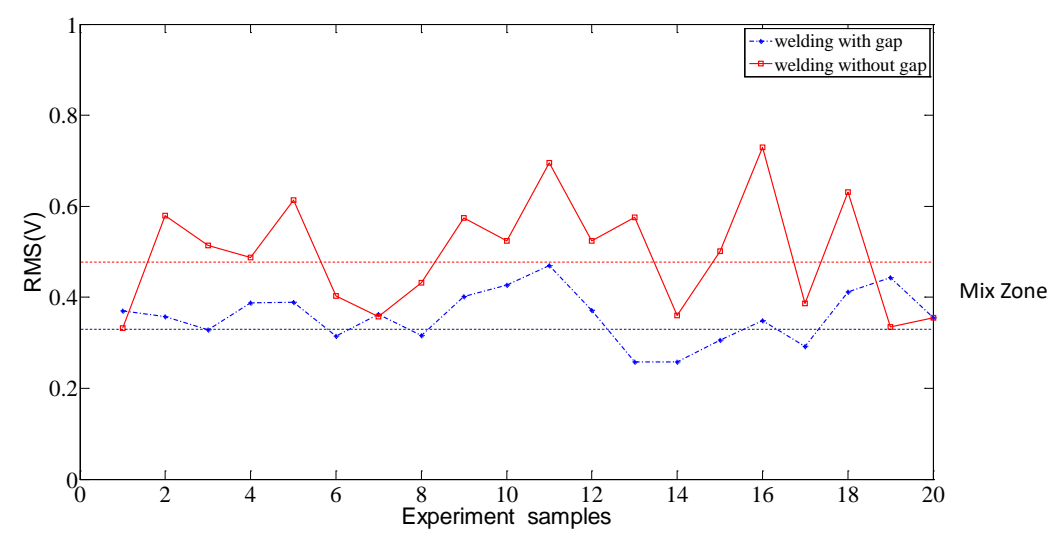

Figure 7. Root-mean-square (RMS) values of full-length sound signals for 20 samples with normal joint strength, and 20 samples with low joint strength.

To analyze time-domain features and extract features closely related to the change of bonding strength, the signal correlated to the entire welding process was divided into eight sections (Figure 8) for feature analysis. The time-domain signals for each selected section are shown in Figure 9. The mixture of obtained signals from both cases can be observed in each section. To extract valuable features for joint-bonding-strength monitoring, RMS and standard deviation (STD) for each section were calculated first. Average values of RMS and STD were obtained from 20 samples for each section (Figure 10). The difference between the cases with high- and low-joint-bonding strengths was observed for both RMS and STD features. Sections 6 and 7 had the highest level of difference. However, on analyzing the level difference of these two features for each sample (Figure 11) in each section, we found that the results were not in agreement with the presented observations in Figure 10. For Sections 2, 6, and 7, presented in Figure 11, the RMS feature level refers to two joint-bonding strengths tangled with each other from sample to sample. This means that these three features cannot be considered proper features for developing a monitoring system, although the average of the RMS feature from all samples had the potential to separate different joint strengths at the feature level. The RMS value of the first section was considered the better choice for identifying low joint strengths. By investigating STD features on the sample base (Figure 12), data from Sections 6 and 7 revealed no promising capabilities in identifying low-joint-bonding strength. Conversely, the STD feature of the first section demonstrated the capability of distinguishing between the high- and low-joint-bonding strength cases. To extract more reliable features from the aforementioned analysis, the STD of each section was divided using RMS values to create a new feature. Values of created STD/RMS ratio features for each section are illustrated in Figure 13. The STD-to-RMS ratio in the second section is a promising feature for identifying the joint-bonding condition.

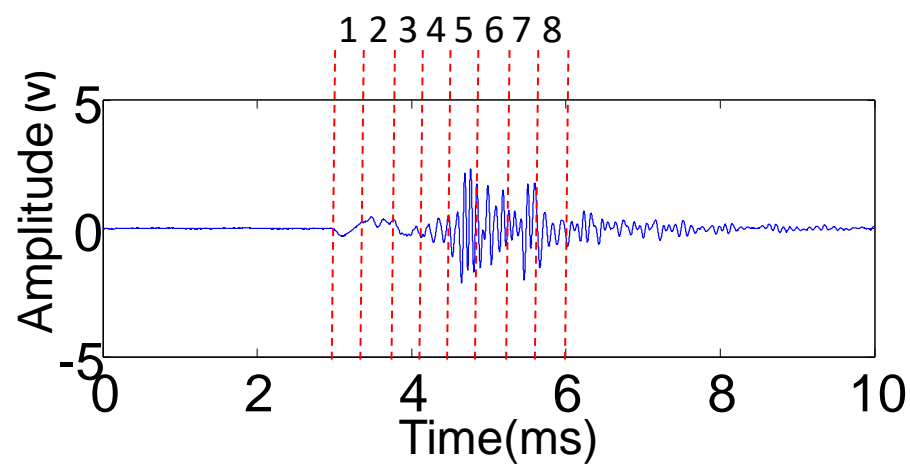

Figure 8. Eight sections of sound signals for analysis. 


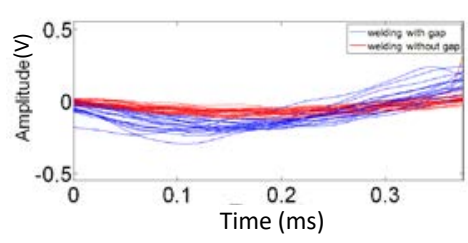

(a)

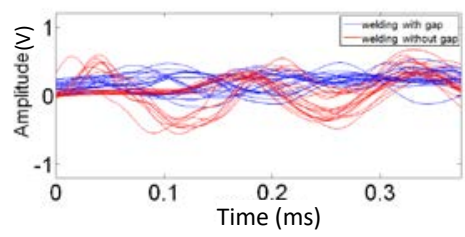

(b)

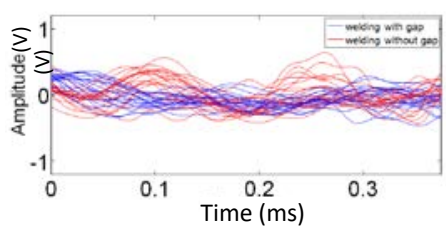

(c)

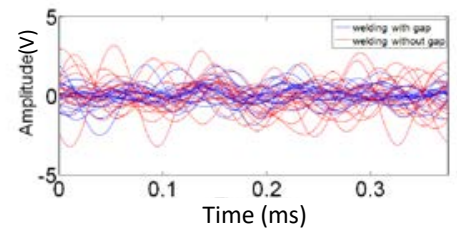

(f)

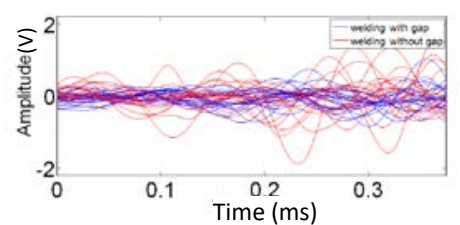

(d)

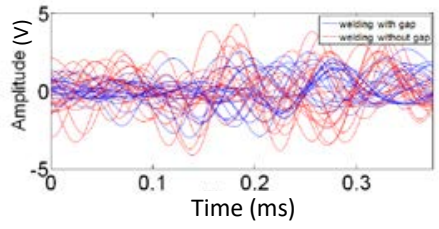

(g)

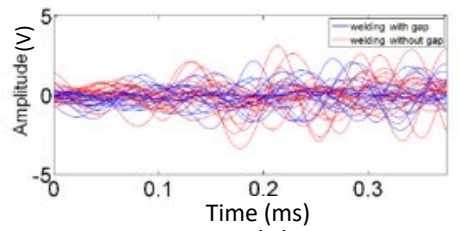

(e)

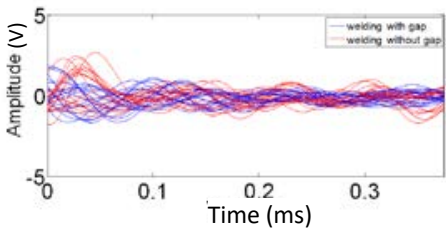

(h)

Figure 9. Time-domain sound signal for each selected section with high- and low-joint-bonding strengths (10 samples for each case): (a) first section; (b) second section; (c) third section; (d) fourth section; (e) fifth section; (f) sixth section; (g) seventh section; (h) eighth section.

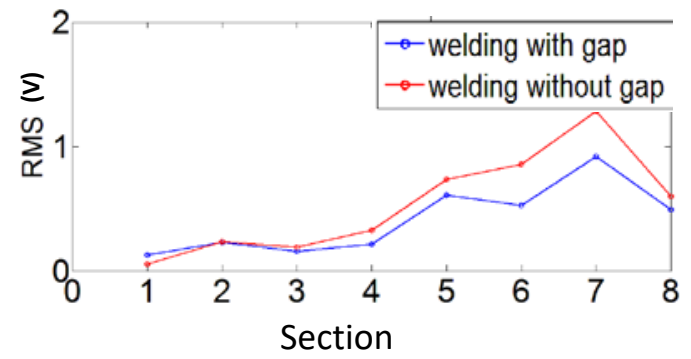

(a)

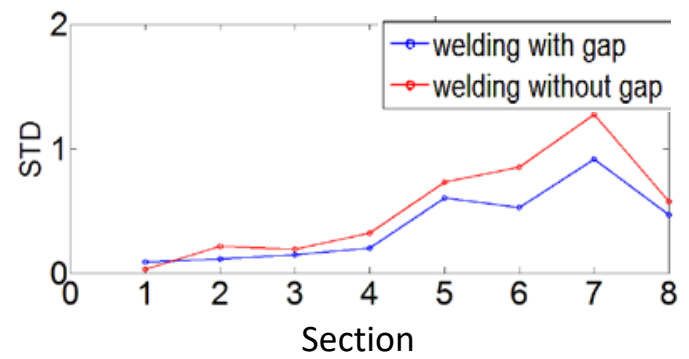

(b)

Figure 10. Average of RMS values and standard deviations (STDs) for 20 samples: (a) RMS and (b) STDs. 


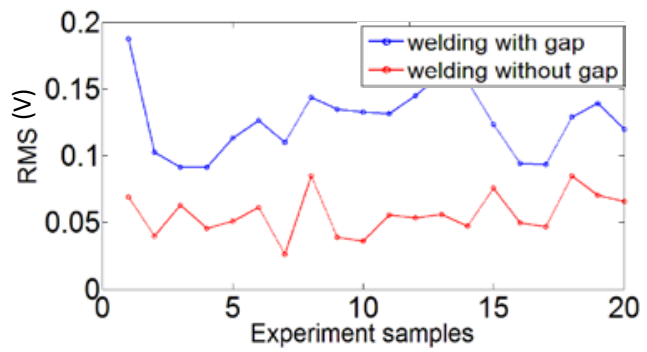

(a)

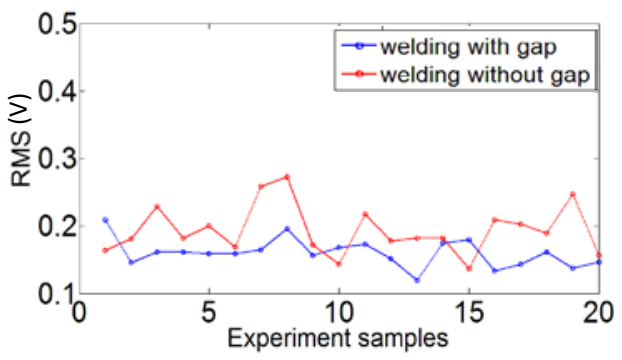

(c)

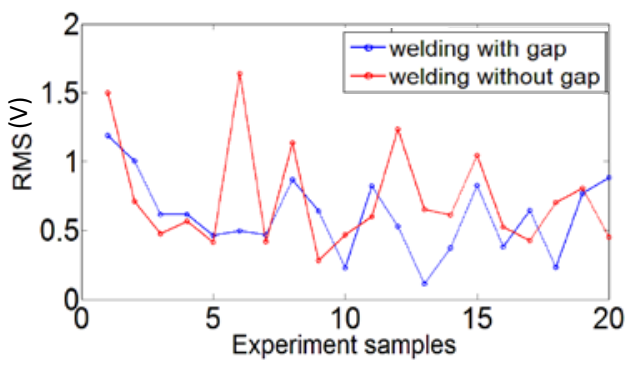

(e)

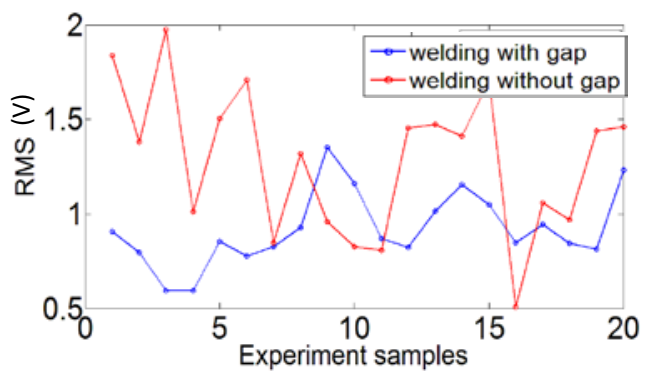

(g)

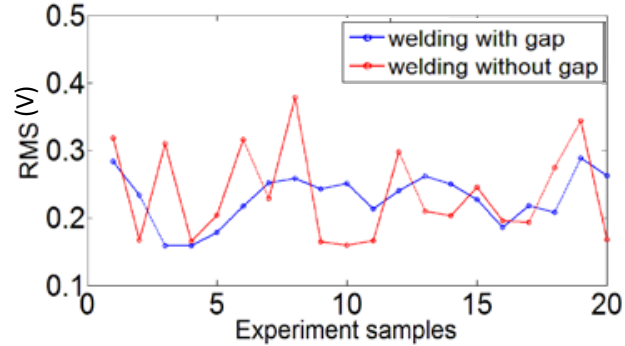

(b)

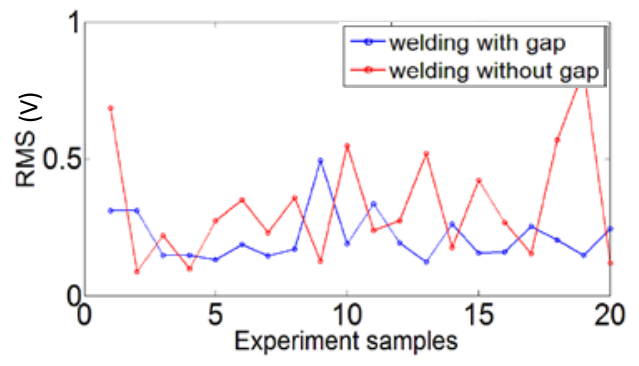

(d)

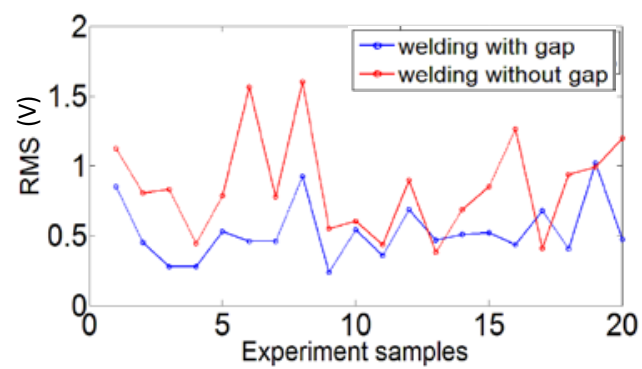

(f)

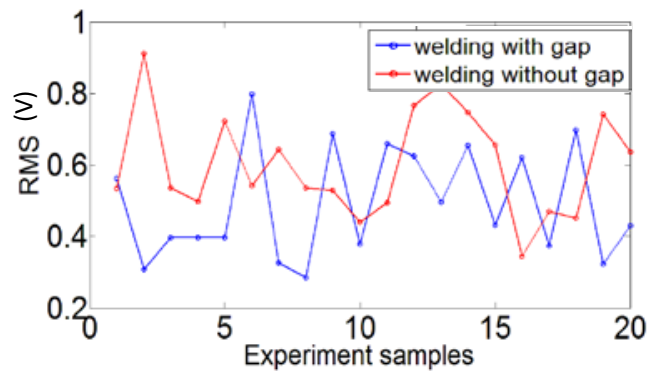

(h)

Figure 11. RMS values for each selected section with high- and low-joint-bonding strengths: (a) first section; (b) second section; (c) third section; (d) fourth section; (e) fifth section; (f) sixth section; (g) seventh section; (h) eighth section. 


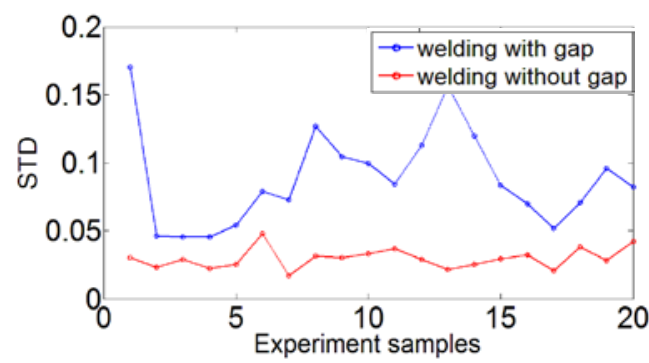

(a)

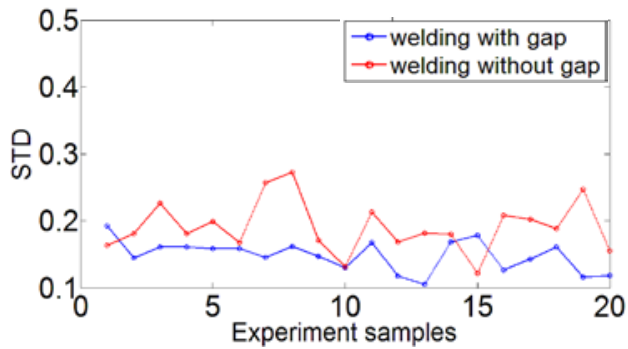

(c)

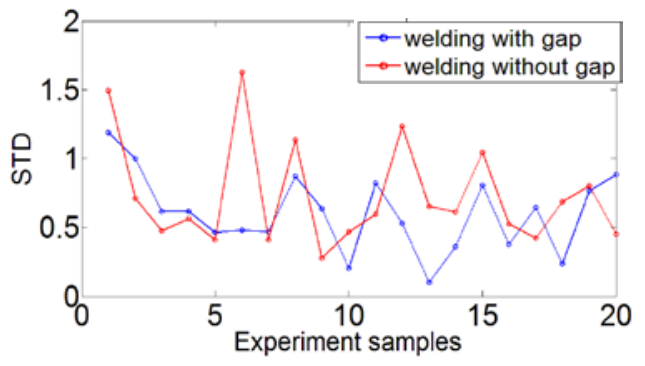

(e)

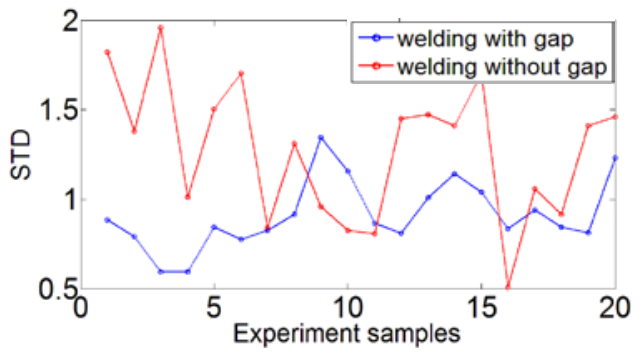

(g)

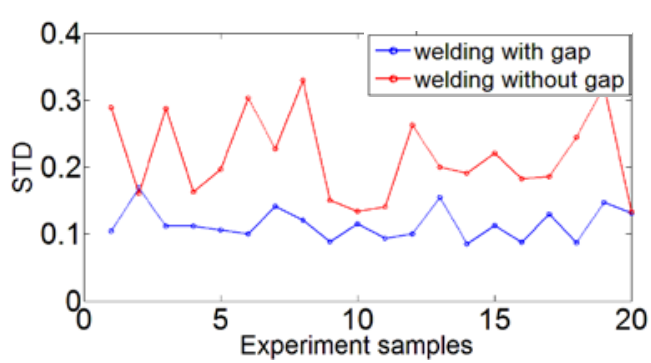

(b)

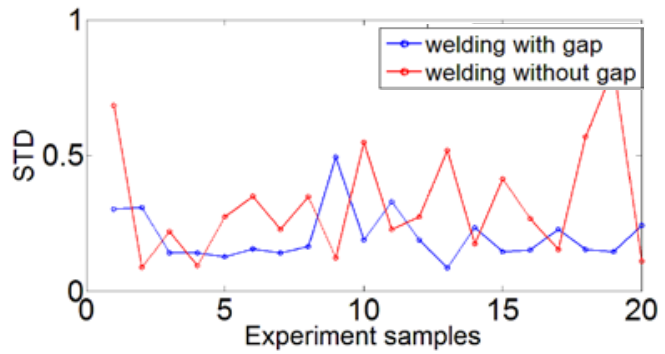

(d)

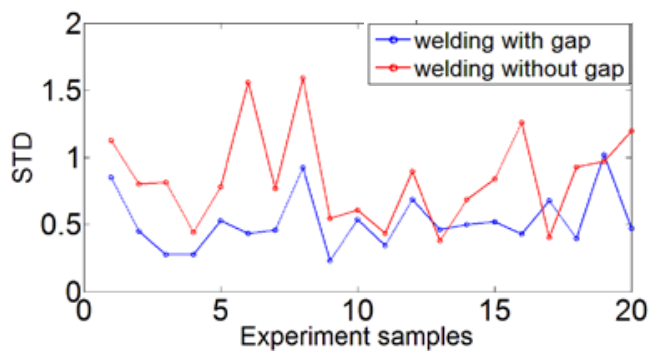

(f)

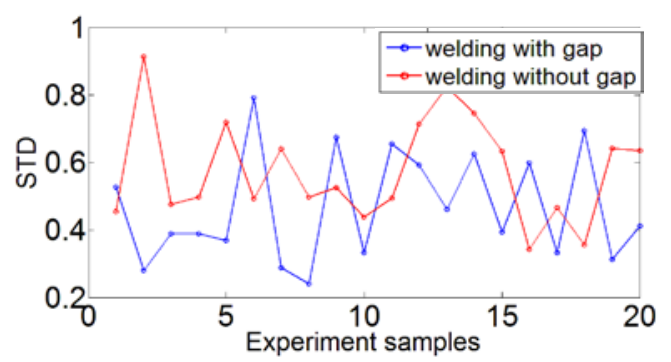

(h)

Figure 12. STDs for each selected section with high- and low-joint-bonding strengths: (a) first section; (b) second section; (c) third section; (d) fourth section; (e) fifth section; (f) sixth section; (g) seventh section; (h) eighth section. 


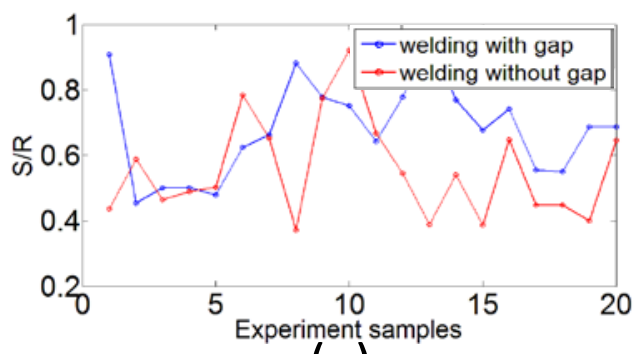

(a)

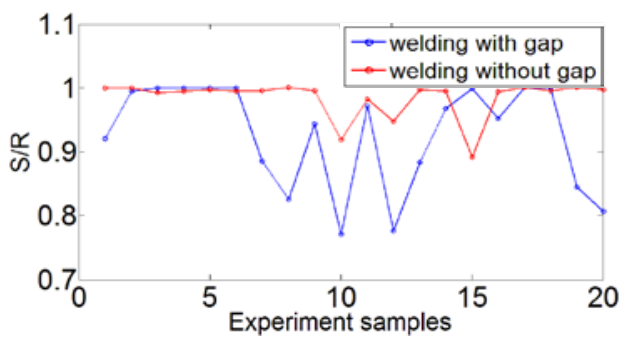

(c)

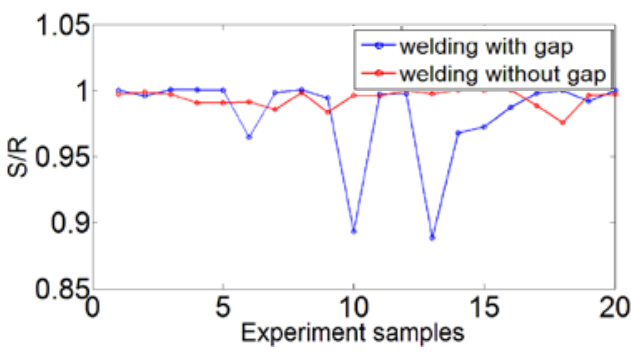

(e)

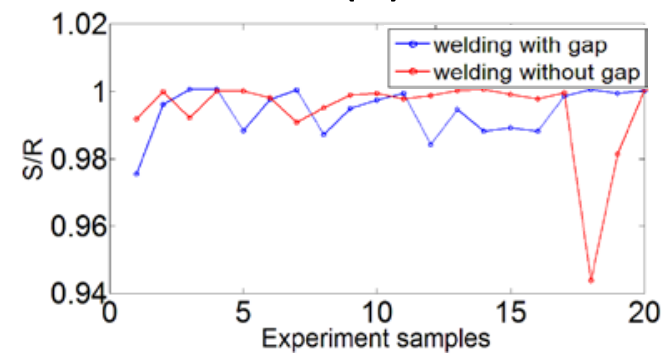

(g)

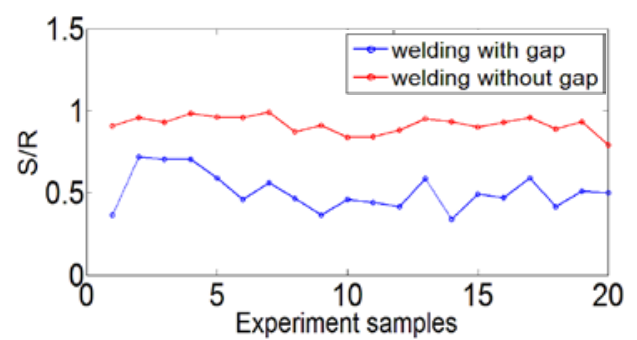

(b)

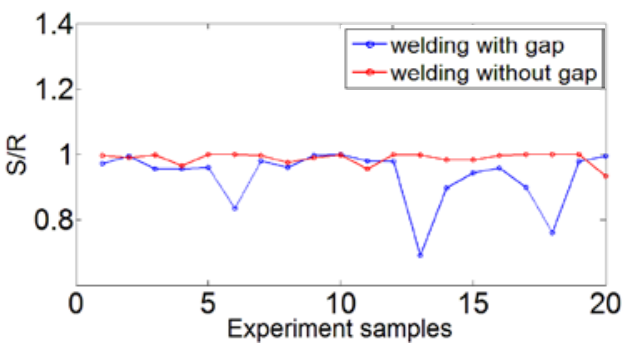

(d)

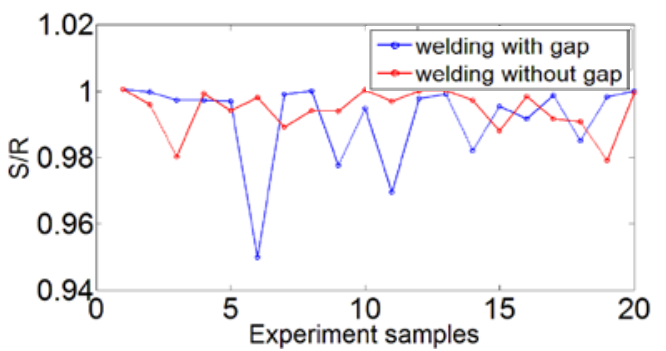

(f)

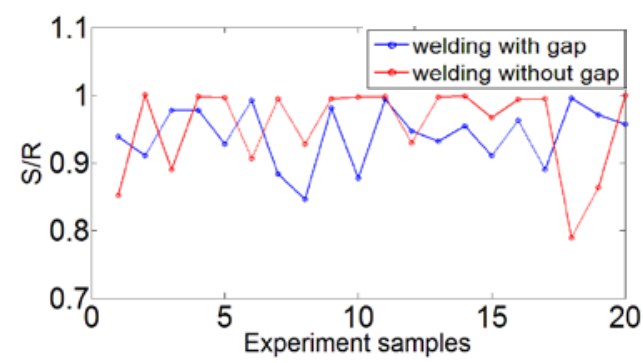

(h)

Figure 13. STD-to-RMS ratio for a selected section with high- and low-joint-bonding strengths: (a) first section; (b) second section; (c) third section; (d) fourth section; (e) fifth section; (f) sixth section; (g) seventh section; (h) eighth section.

To quantify the capability level of each feature for identifying the joint condition, scatter index $J$ was calculated on the basis of the ratio of between-class to in-class distribution for the three aforementioned features for each section. Results presented in Figure 14 showed that RMS values and STDs in the first section, and the STD-to-RMS ratio in the second section had the highest $J$ values and could be considered optimal candidates as inputs for the classifier. To evaluate selected features for monitoring the low joint strength caused by the gap generated between metal sheets in laser microlap welding, an HMM-based classifier was implemented with selected features [17]. Observation sequence levels are listed in Table 3. The model was developed using 10 sets of data that were obtained from 10 samples and evaluated by 10 other sets of data. A 100\% classification rate was obtained with the three selected features. 

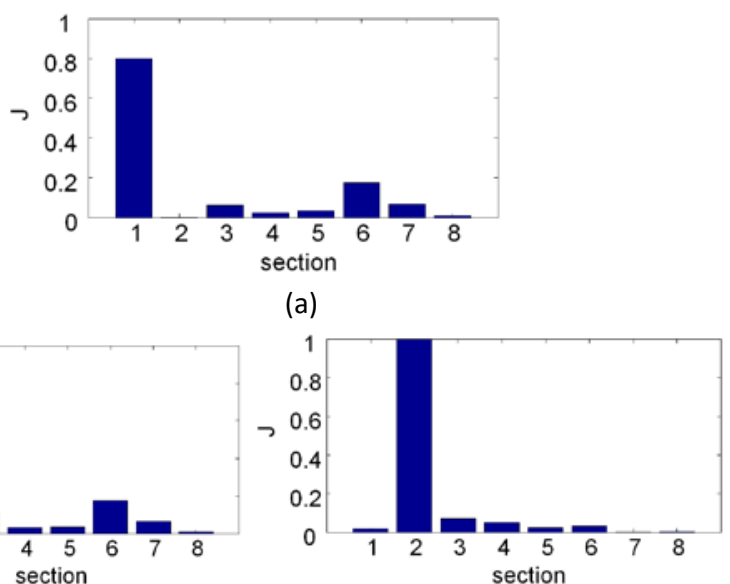

(b)

(c)

Figure 14. Classification index for each section: (a) RMS value; (b) STD; (c) STD-to-RMS ratio.

Table 3. Selected features for hidden Markov model (HMM)-based classifier.

\begin{tabular}{|c|c|c|c|}
\hline \multicolumn{2}{|c|}{ Features } & \multicolumn{2}{|c|}{ Observation Level Assignment } \\
\hline \multirow{8}{*}{ Section 1} & \multirow{3}{*}{ RMS } & $<0.09$ & 1 \\
\hline & & $0.09-0.18$ & 2 \\
\hline & & $>0.18$ & 3 \\
\hline & \multirow{5}{*}{ Standard Deviation } & $<0.04$ & 1 \\
\hline & & $0.04-0.08$ & 2 \\
\hline & & $0.08-0.12$ & 3 \\
\hline & & $0.12-0.16$ & 4 \\
\hline & & $0.16-0.2$ & 5 \\
\hline \multirow{2}{*}{ Section 2} & \multirow{2}{*}{ Ratio of RMS to STD } & $<0.75$ & 1 \\
\hline & & $>0.75$ & 2 \\
\hline
\end{tabular}

\section{Conclusions}

In this study, analysis of the correlation between sound signals and joint strength in laser lap microwelding was conducted by dividing sound signals into eight sections. Results revealed that RMS and STD values of the first section of signals, and the STD-to-RMS ratio of the second section of signals in the welding process have promising potential in identifying low-strength joints. A $100 \%$ classification rate was obtained when normal and low-strength joints were monitored using an HMM-based classifier with selected features to verify the performance of the three selected features.

Author Contributions: B.-S.K. contributes to the conduction of experiments, signal processing, and writing. M.-C.L. contributes to the conceptualization, methodology, development, signal analysis, and writing. All authors have read and agreed to the published version of the manuscript.

Funding: The conducted experiments in this study were supported by the Geniray Technology Corporation in New Taipei, Taiwan. This research also partially received financial supports from Ministry of Science and Technology in Taiwan. Grant number: [MOST 104-2221-E-005-019 -].

Conflicts of Interest: The authors declare no conflict of interest. 


\section{References}

1. Shao, J.; Yan, Y. Review of Techniques for On-line Monitoring and Inspection of Laser Welding. J. Phys. Conf. Ser. 2005, 15, 101-107. [CrossRef]

2. Smith, E.T. Monitoring Laser Weld Quality Using Acoustic Signals. Ph.D. Thesis, University of Michigan, Ann Arbor, MI, USA, 1999.

3. Sun, A.S. Multiple Sensor Monitoring of Laser Welding. Ph.D. Thesis, University of Michigan, Ann Arbor, MI, USA, 2000.

4. Shimada, W.; Ohmine, M.; Hoshinouchi, S.; Kobayashi, M. Study on In-Process Assessment of Joint Efficiency in the Laser Welding Process. In Proceedings of the International Symposium of the Japan Welding Society, Osaka, Japan, 24-26 November 1982; pp. 175-180.

5. Mombo-Caristan, J.C.; Koch, M.; Prange, W. Seam Geometry Monitoring for Tailored Welded Blanks. LIA 1992, 74, 123-132.

6. Gu, H.; Duley, W.W. A Statistical Approach to Acoustic Monitoring of Laser Welding. J. Phys. Appl. Phys. 1996, 29, 556-560. [CrossRef]

7. Grad, L.; Grum, J.; Polajnar, I.; Slabe, J.M. Feasibility Study of Acoustic Signals for On-Line Monitoring in Short Circuit Gas Metal Arc Welding. Int. J. Mach. Tools Manuf. 2004, 44, 555-561. [CrossRef]

8. Nava-Rüdiger, E.; Houlot, M. Integration of Real Time Quality Control Systems in a Welding Process. J. Laser Appl. 1997, 9, 95-102.

9. Luo, H.; Zeng, H.; Hu, L.; Hu, X.; Zhou, Z. Application of Artificial Neural Network in Laser Welding Defect Diagnosis. J. Mater. Process. Technol. 2005, 170, 403-411. [CrossRef]

10. Matteson, A.; Morris, R.; Tate, R. Real-Time GMAW Quality classification using an artificial neural network with airborne acoustic signals as inputs. In Proceedings of the 12th International Conference on Offshore Mechanics and Arctic Engineering III-A, Glasgow, Scotland, 20-24 June 1993; pp. 273-278.

11. Lin, R.H.; Fischer, G.W. An On-Line Arc Welding Quality Monitor and Process Control System. In Proceedings of the IEEE Conference on Industrial Automation and Control Emerging Technology Applications, Taipei, Taiwan, 22-27 May 1995; pp. 22-29.

12. Farson, D.; Hillsley, K.; Sames, J.; Young, R. Frequency-Time Characteristics of Air-Borne Signals from Laser Welds. J. Laser Appl. 1996, 8, 33-42. [CrossRef]

13. Jin, X.; Li, L.; Zhang, Y. A Study on Fresnel Absorption and Reflections in the Keyhole in Deep Penetration Laser Welding. J. Phys. D Appl. Phys. 2002, 35, 2304-2310. [CrossRef]

14. Huang, W.; Kovacevic, R. Noise Reduction During Acoustic Monitoring of Laser Welding of High-Strength Steels. In Proceedings of the ASME 2009 International Manufacturing Science and Engineering Conference, West Lafayette, IN, USA, 4-7 October 2009; pp. 763-770, (MSEC2009-84080).

15. Chien, K. Applications of Acoustic Emission and Sound Signal for Welding Defect Monitoring in Nd:YAG Laser Thin Plate Butt Welding. Master's Thesis, National Chung Hsing University, Taichung City, Taiwan, 2012.

16. Hsieh, W.; Lu, M.; Chiou, S. Application of backpropagation neural network for spindle vibration-based tool wear monitoring in micro-milling. Int. J. Adv. Manuf. Technol. 2012, 61, 53-61. [CrossRef]

17. Wang, C.Y. The Development of Tool Condition Monitoring in the Millingof Inconel 718 by Recurrent HMMs. Master's Thesis, National Chung Hsing University, Taichung City, Taiwan, 2018.

(C) 2020 by the authors. Licensee MDPI, Basel, Switzerland. This article is an open access article distributed under the terms and conditions of the Creative Commons Attribution (CC BY) license (http://creativecommons.org/licenses/by/4.0/). 